\title{
EN LA PUERTA DEL INFIERNO. PROXIMIDAD, TENSIONES Y ESCENARIOS DIFÍCILES EN MEDIO DEL CAMBIO CLIMÁTICO
}

\section{At the gate of hell. Proximity, tensions and difficult scenarios amid climate change}

Franklin Américo CanazaChoque $^{1}$

CONCYTEC

PERÚ

leo_123fa@hotmail.com

https://orcid.org/oooo-0oo2-19296054

\author{
Lucio Bernardo \\ Condori-Pilco $^{2}$ \\ UNIVERSIDAD NACIONAL DEL \\ ALTIPLANO \\ PERÚ \\ lcondorip@unap.edu.pe \\ https://orcid.org/oooo- \\ 0002-7629-7395
}

\author{
Jaen Paúl Peralta- \\ Cabrera 3 \\ UNIVERSIDAD NACIONAL DEL ALTIPLANO \\ PERÚ \\ jpperalta@cepreuna.edu.pe \\ https://orcid.org/oooo-0002- \\ 0403-676X
}

\author{
Rudy Oswaldo Dávila-Quispe ${ }^{4}$ \\ UNIVERSIDAD NACIONAL DEL ALTIPLANO \\ PERÚ \\ 70376108@cepreuna.edu.pe \\ https://orcid.org/oooo-0002-2208-6234
}

DOI: https://doi.org/10.35622/j.rr.2021.03.002

Recibido: 06-I-2021 / Aceptado: 10-III-2021 / Actualizado: 17-III-2021

\section{Resumen}

Degradación de la tierra, reducción glaciar, desastres naturales extremos, desertificación a escala, inundaciones, incendios forestales, ciclones tropicales, flujos de gases de efecto invernadero, incremento de enfermedades, conflictos hídrico-territoriales, desplazados ambientales, sobrecalentamiento terrestre-oceánico, hambre e inseguridad alimentaria internacional, entre otros. Todos y cada uno, sin duda, pueden tener un escenario de daño desmedido que incluso, pudiesen estar relacionados por un nodo principal que las ocasiona,

1 Docente-Investigador Social del Consejo Nacional de Ciencia, Tecnología e Innovación Tecnológica [CONCYTEC].

${ }^{2}$ Licenciado en Educación Secundaria con Mención en Ciencias Sociales, Magíster Scientiae en Administración de la Educación por la Universidad Nacional del Altiplano de Puno [UNAP].

3 Licenciado en Ciencias de la Educación, Maestrando en Investigación y Docencia Universitaria en la Unidad de Postgrado de la Facultad de Ciencias Sociales. Estudios concluidos en Maestría en Educación con Mención en Didáctica de las Ciencias Sociales de la Escuela de Posgrado de la Universidad Nacional del Altiplano de Puno [UNAP].

4 Licenciado en Ciencias de la Educación, Maestrando en Didáctica de las Ciencias Sociales en la Escuela de Posgrado de la Universidad Nacional del Altiplano de Puno [UNAP]. 
el que justamente, tiene una poderosa rectoría social, normativa, política-económica y certera explicación profunda: los impactos deprimentes del cambio climático en el terreno global. En esos denominadores, este breve estudio forma parte de un gran inventario de temas delimitados sobre la problemática ambiental en el que se busca, preponderantemente, describir el desastre próximo y los daños climáticos globales en tiempos en el que las opciones muestran su propio agotamiento. Pues, todo indica que es posible que esperemos el desastre el día de mañana.

Palabras Clave: COVID-19, cambio climático, desarrollo sostenible, medio ambiente.

\begin{abstract}
Land degradation, glacial reduction, extreme natural disasters, scale desertification, floods, forest fires, tropical cyclones, greenhouse gas flows, increase in diseases, water-territorial conflicts, environmental displacement, land-ocean overheating, hunger and international food insecurity, among others. Each and everyone, without a doubt, may have a scenario of excessive damage that could even be related by a main node that causes them, the one that precisely has a powerful social, normative, political-economic leadership and an accurate deep explanation: the depressing impacts of climate change on the global terrain. In these denominators, this brief study is part of a large inventory of delimited topics on environmental problems in which it is sought, predominantly, to describe the upcoming disaster and global climate damage at a time when options show their own exhaustion. Then, everything indicates that we may expect disaster tomorrow.
\end{abstract}

Keyword: COVID-19, climate change, sustainable development, environment.

\title{
INTRODUCCIÓN
}

Detener, paliar o neutralizar el Cambio Climático (CC) no es una opción. Es la única que existe. En medio de un escenario de poderosas fricciones de problemas globales que se entremezclan de una manera que resulta difícil trazar una ruta puntual que permita planear un desarrollo internacional sostenido e inclusivo de largo plazo. Pueden también, del mismo modo, liberarse otras brutales realidades en diferentes partes del mundo que, debido a su gravedad, son imputaciones en todas las escalas geográficas. Entre ellos destaca, de acuerdo al informe desprendido por una de las mayores agencias de las Naciones Unidas para la Agricultura y la Alimentación (FAO) y destinada en ponerle fin al hambre, es que, este último propósito diagonal de estado de seguridad alimentaria y nutrición global podrían seguir estando más allá del 2030 como grandes rectores de un desafío inacabado e intensificado por los impactos adversos de la pandemia del COVID-19 (FAO, 2020).

En la misma dirección de agravar el equilibrio mundial, pero con velocidades diferentes en cada zona del planeta. La desigualdad global nota ser otro chocante problema del presente siglo. No es nada extraño, que este mismo crecimiento desigual de la época contemporánea ponga en peligro el desarrollo de metas internacionales. Así, erradicar o reducirla, pueden ser las únicas alternativas en la arena política. No dejar a nadie atrás, es con buena razón, el grito 
de guerra de la Agenda 2030 para el Desarrollo Sostenible. No obstante, de acuerdo al balance actual, el extraordinario crecimiento económico del que se refiere y de las generalizadas medidas en promover el estado de bienestar social, no han logrado durante las últimas décadas cerrar las divisorias profundas dentro y entre países. Así, por ejemplo, las actuales propensiones de innovación, $\mathrm{CC}$, urbanización y migración internacional pueden un tanto ampliar las oportunidades de transformación y desarrollo en algunos; pero, en la gran mayoría de regiones de estructuras débiles, pueden exacerbar disparidades y divisiones inacabables, que, en gran medida, determinen nuestro futuro común (United Nations Department of Economic and Social Affairs [UNDESA], 2020).

Otra megatendencia que viene opacando la proximidad, es en efecto, una amenaza mundial aerotransportada, no del todo invisible, pero que permanente sigue siendo ignorada en muchas partes del mundo: la contaminación del aire. Entre una explosión de datos, en el 2019 la contaminación atmosférica se interpuso como el cuarto factor de riesgo principal de muerte a nivel planetario. Un valor que se tradujo aproximadamente en 6,75 millones de muertes prematuras y 213 millones de años de vida sana perdidos. Dado que respirar aire de mala calidad, representa sin duda, uno de los peligros más altos para la salud humana y del medio ambiente (Health Effects Institute, 2020). De esto, el actual Director General de la Organización Mundial de la Salud (OMS), Tedros Adhanom Ghebreyesus, refirió en el 2018 la poderosa argumentación de que, si no adoptamos medidas urgentes contra este enemigo global, toda idea que devenga del y sobre el Desarrollo Sostenible (DS) será una mera imaginación, ya que, el aire, cuando es y está contaminado, amenaza a todos, especialmente, a quienes conforman las grandes plataformas o pedestales de pobreza y marginación (OMS, 2018c).

Entre el drama de un terror interminable, al menos durante los últimos 10 años se ha tenido un aproximado de 100 millones de personas que se han visto forzadas a buscar un refugio dentro o fuera de las fronteras de su país. Este desplazamiento de personas a nivel global, sin lugar a dudas, ocuparon la atención en todas parte del mundo, además de tener un lugar destacado en la agenda internacional sobre una crisis sin precedentes que incluiría múltiples causales, como por ejemplo, el estallido del conflicto armado en Siria, la emergencia humanitaria que siguió a la independencia de Sudan del Sur, la brutalidad del conflicto y de una zona minada en Ucrania, el estado de alerta máxima por la llegada de refugiados y migrantes a Europa, el flujo intensivo de refugiados rohingya de Myanmar a Bangladesh, el éxodo masivo de venezolanos a tierras extrañas, la violencia indiscriminada en la región del Sahel, las nuevas tensiones sobre seguridad en Iraq, Afganistán, Libia y Somalia, el desplazamiento interno en Etiopía, la lucha y violencia en la República Democrática del Congo y la situación trágica en Yemen (Alto Comisionado de las Naciones Unidas para los Refugiados [ACNUR], 2020). Todos y cada uno de estos problemas de fuga y escape pueden indicar que el estado actual de los derechos humanos y el orden público mundial están siendo gravemente dañados por la misma presión y tensidad del momento, la persecución, el conflicto, la violencia y la alteración de fuerzas climáticas que no dejan de apartarse de todos estos problemas clave. 
En ese mismo sentido y en el mismo episodio de voluminosos problemas transversales, este breve estudio forma parte de un gran catálogo de temáticas delimitadas sobre la problemática ambiental en el que se busca, preferentemente, describir el desastre próximo y los daños climáticos globales en tiempos en el que las opciones muestran su propio agotamiento. Pues, todo indica que es posible que esperemos la catástrofe el día de mañana.

\section{METODOLOGÍA}

En el marco de los estudios de investigación teórico documental, el trabajo, desde un primer momento y en la búsqueda de solidificar los argumentos necesarios para delimitar los alcances del problema, se interpone más fuertemente un rastreo bibliográfico de archivos digitales con factor de impacto. En ese mismo procedimiento, una vez administrado la documentación adecuada, se desarrolló la técnica del fichaje con el propósito de recolectar, ordenar ideas y almacenar información acerca de los impactos desprendidos del CC en las proximidades del futuro. Igualmente, la investigación revistió un repertorio de 34 referentes bibliográficos enmarcados por terminaciones clave como CC, DS, Calentamiento Global (CG), contaminación ambiental, medio ambiente, entre otros. Para finalizar, de extremo a extremo se recurrió a los métodos analíticos, reflexivos e inferenciales con el objeto de consolidar un desarrollo temático, a la vez de empoderar una conclusión de lo más pertinente (CanazaChoque, Supo, Ruelas y Yabar, 2020; Canaza-Choque, 2020a; 2020c; 2021a; 2021b; 2021c; Huanca-Arohuanca, 2020a; 2020b; 2021).

\section{DESARROLLO}

\section{Proximidades, desastres y daños climáticos globales}

2030 será el marcador para ver si efectivamente se han llegado a cumplir con los 17 objetivos trazados para el milenio en rotativa, todos presentados en la denominada Agenda para el Desarrollo Sostenible 2030 como un desafío planetario de tres esferas por atender: las personas, el planeta y la prosperidad. Una visión integral adoptada en 2015 en la cumbre de las Naciones Unidas (UN) que busca en el sentido amplio del término acabar con las proyecciones coercitivas del CC sobre estos planes globales (Aznar y Barrón, 2017). En efecto, si los propósitos propuestos antes y después, para el 2050 no lograsen lo prometido para un aproximado de 10 mil millones de humanos que habitarían el planeta hasta ese entonces, el riesgo rebasaría los límites planetarios, asumiendo cambios catastróficos en el sistema terrestre y de todo aquello que lo ensambla (Rivera, 2019).

Por ello, la actual economía mundial requiere apostar por la descarbonización del cíclico modelo económico de poder-mundo, que no solo afectan tóxicamente a otras dimensiones comparadas con la salud pública internacional; sino también, rebasan en su mayoría a los objetivos globales del DS. En esa intensión, asumirla como desafío nodal a pesar de su abrumadora dependencia, obliga radicalmente a reorientar en profundidad y de toda estructura el modo y estilo de vida supeditado a una alta composición de matriz energética configurada por materiales fósiles que, 
en su mal y extendido uso pueden invitar a que se supere la línea roja, y que finalmente, conduzcan a asistir a un banquete de consecuencias catastróficas poco agradables para el benefactor humano (Sanabria, 2018; Canaza-Choque, 2020b).

De esto, según el informe que se maneja por parte de la OMS (2018a), dicha institución global, al realizar una evaluación sobre las posibles repercusiones sanitarias que anualmente causarían el CC, resume que entre 2030 y 2050 se tendría 95.000 defunciones por desnutrición infantil; 60.000 por paludismo; 48.000 por diarrea; y 38.000 por exposición de personas ancianas al calor. Al mismo tiempo, entre una alerta emitida por la misma agencia, se expuso que el $90 \%$ de la población mundial actualmente respira aire contaminado. Ilustrando así, una muestra de 4,2 millones de muertes relacionadas con la mala calidad del aire durante el 2016 (OMS, 2018b).

Debido a esto, reducir o acabar con las emanaciones de Gases de Efecto Invernadero (GEI) representa uno de los mayores desafíos de la política exterior climática. Pues, contrariamente a ello, es decir, su elusión o abstenerse, puede explicar un pronóstico para el 2100, y es que resulta que poco más de la mitad de la población mundial se vería afectada con desoladoras sequías, enormes olas de calor (Mora et al., 2018), deshielos, lluvias e inundaciones, mega tormentas, enfermedades, inseguridad alimentaria, extinciones y estragos en la biodiversidad global (Fernández, 2019).

En tal sentido, un punto inquebrantable es que si se continúa usando en una dimensión similar o más todos los combustibles fósiles en la tierra, para el 2300, el CG acabaría de por sí con toda la especie sobre el plano terrestre (Zimmer, 2018). Para reforzar lo anterior, el nivel de confianza del quinto informe de evaluación presentado por el Panel Intergubernamental del Cambio Climático (IPCC, 2014) está seguro que, de todos modos, los cambios irreversibles y abruptos irán más allá del 2100. Inclusive, todos los posibles escenarios que puedan fijarse o ser recreados serán afectados a medida que aumente la magnitud del CG.

Esto, mecánicamente se traduce en fuertes impactos degradatorios para el orden medioambiental, social y económico. Pues, la magnitud del aumento de la temperatura media, causado principalmente por la alta emisión de dióxido de carbono $(\mathrm{CO} 2)$ o forzamientos extremos de GEI, son dominantes de la intensificación y del daño provocado por el CC (Petersson, Marrero y Taboada, 2010). Sin duda, este último, puede irradiarse en una amplia gama de acontecimientos que van desde el incremento de flujos migratorios, inseguridad alimentaria, escasez hídrica, deterioro de la salud, hasta incluso, más allá de todo esto, provocar conflictos bélicos, motivaciones geopolíticas de disputa e intensificar negativamente en el bienestar de los seres humanos (Cuevas y Preciado, 2018).

No obstante, si bien la distribución de impactos resulta ser diferenciado, los episodios extremos acabarían minando no solo únicamente los sistemas humanos y gestionados (producción de alimentos, medios de subsistencia, salud, actividades económicas), sino que además, llegaría a alterar globalmente el comportamiento natural y las funciones regulares de los sistemas físicos 
(glaciares, hielo, ríos, lagos, nivel del mar) y biológicos (ecosistemas terrestres y marinos) (IPCC, 2014).

De esta manera, el CC se ha convertido en lo que va de los años en una de las mayores amenazas para la existencia de microclimas, mamíferos, insectos, aves, peces y plantas, que en todo caso, han tenido que evolucionar a ritmos poco divisados para sobrevivir (Plumer, 2019). E incluso, cuando se pensaba que la adaptación sería el camino de la naturaleza a seguir, la velocidad del CC llegó a superar cualquier otra forma de capacidad adaptativa disponible (García, 2017). Pues, una muestra de su mordacidad en los animales, es que este, los empuja hasta el límite: adaptarse, marcharse o perecer, son algunas opciones al que los escenarios con las que se ha desplazado el CC los pone al borde de la extinción parcial o total de la especie, o en el peor de los casos, a un escenario al que los científicos catalogan como coextinciones, es decir, donde un organismo desaparece de la faz de la tierra porque depende de otra especie que ha quedado condenada a un peligro de extinción irrevocable (National Geographic, 2018; Guasch, 2019).

De hecho, uno de los históricos informes que realizó la Plataforma Intergubernamental de Ciencia y Política sobre Biodiversidad y Servicios de Ecosistemas (IPBES), es que revela que el peligro de extinción, al que se ubican un millón de especies de animales y plantas, podrían llegar a desaparecer en tan solo algunas décadas (UN, 2019).

En este mismo terreno, el IPCC (2015) tiene las cosas claras. Pues, este recomienda dos medidas parcialmente complementarias que bien podrían amortiguar las causas o consecuencias del fenómeno global. La primera, de adaptación o ajuste climático real o proyectado, una que gravita en reducir la vulnerabilidad y exposición a la variabilidad climática, destinando todas sus fuerzas en promover sinergias con el desarrollo y en la capacidad de reducir, moldear o evitar los daños que se emanen de esta. Mientras que la segunda, el de la mitigación, es una estrategia dirigida a acabar con las causas que la originan, de tal manera, que se encamina a reducir las concentraciones de GEI mundiales y de potenciar la construcción de sumideros de carbono.

Sobre esto, una de las recomendaciones por el nivel de escala global y de alcance regional acerca de la determinación más efectiva de estas medidas, es que los países en desarrollo inviertan, más precisamente por el uso intensivo de políticas de adaptación reactiva, debido a que no disponen de recursos propios para hacer frente a los impactos proyectados por el CC. En tanto que, los países desarrollados, por su volumen y magnitud económica, tendrían más bien que desarrollar, principalmente, acciones y modelos de mitigación contra el CC (Hasbún-Mancilla, Aldunce-Ide, Blanco-Wells y Browne-Sartori, 2017). A este último, según el Acuerdo de París (AP) de 2016, estos también deberían, no solo de detener la industria del carbón global y de alcanzar las emisiones cero hasta la segunda mitad del siglo, sino que además, están en el fallo de apoyar a los países en vías de desarrollo en la búsqueda de una sociedad menos contaminante y más resiliente a los efectos contraproducentes del CC (Cuevas y Preciado, 2018).

En afinidad, si bien el AP busca alcanzar el propósito global de mantener la temperatura media por debajo de los $2{ }^{\circ} \mathrm{C}$, de neutralizar el carbono hacia 2050 y de no ceder ante las amenazas del 
CC. Dicho pacto internacional, también exhorta a que las partes vinculantes desarrollen medidas de prevención y que adopten maneras para conservar y acrecentar los sumideros y reservorios de GEI para los siguientes años (Sarmiento, 2018). Dentro de ese plan, la lucha contra el CG y de daño emergente deliberan dos respuestas estratégicas a abordarse: adaptación y mitigación. Para algunos (Castillo, 2016; Hasbún-Mancilla et al., 2017), ambas deben de ir en y con la misma correlación de poder reducir la gravedad del CC, pero instalando una mayor catadura en la segunda. E inclusive, prescindir en este último, le corresponde más radicalmente a los países desarrollados, mientras que los esfuerzos adaptativos, por lo pronto, importa a los países en vías de desarrollo, esto, debido a sus características de réplica e impacto al CC.

De esta manera, el $\mathrm{CC}$ es, desde cualquier ángulo del que se vea, un desafío rector letal y fenómeno global conocido de sobra que exige todo tipo de respuesta que implique acabar con este escenario difícil. El gran proceso está definido, sin duda, por un repertorio de daños masificadores y riesgos en el orden internacional. Efectos y presiones que no solamente se entremezclan y refuerzan mutuamente con el propósito de desequilibrar toda articulación posible entre los Estados para hacerle frente, sino que además, dada su magnitud de impacto y del alcance desmedido, no puede negarse que aumenta una mayor probabilidad de que las respuestas mitigadoras $\mathrm{y} / \mathrm{o}$ adaptativas recreadas durante los últimos tiempos sean insuficientes en poder detener eficazmente la fricción de este problema hegemónico.

\section{CONCLUSIONES}

De lo ya conocido, podemos decir que, el CC es el desastre que acompaña la ruta de nuestros días. Sobre esto, no hay duda. Pues, los impactos y escenarios recreados constituyen, por un lado, el tenor de todos los tiempos; y, por otro lado, esta misma hostilidad se convierte en el mayor desafío global que exige un esfuerzo-respuesta de remodelar el modelo económico imperante, social, distributivo y de innovación que permita subsanar el pasado, reparar el presente y transitar de un estado de depredación internacional, a un estado de DS e inclusivo entre todas las regiones del planeta.

Lo antes mencionado, de por sí ya establece una cadena de altas soluciones que repelen los efectos negativos del CC. No obstante, las respuestas desprendidas han sido menores a la interrogante del impacto. En ese sentido, si bien el estado de calamidad pública y de daños globales reducen la posibilidad de detener su avance mortífero. Esto también demanda, de todas formas, tomarse el asunto con mucha seriedad y una enérgica capacidad decisoria. Así, el plano perfecto para su tratamiento sigue estando en la agenda y de las decisiones de la política internacional. Pues, políticamente, serán los Estados poderosos quienes decidan en mayor fuerza si continuamos o no, en el camino de ir hacia el abismo de nuestros días.

\section{REFERENCIAS BIBLIOGRÁFICAS}

Alto Comisionado de las Naciones Unidas para los Refugiados [ACNUR]. (2020). Tendencias globales. Desplazamiento forzado en 2019. Suiza: ACNUR. 
Aznar, P., \& Barrón, Á. (2017). El desarrollo humano sostenible: un compromiso educativo. Teor. Educ., 29(1), 25-53. https://doi.org/10.14201/teoredu20172912553

Canaza-Choque, F. A. (2020a). Educación superior en la cuarentena global: disrupciones y transiciones. Revista Digital de Investigación en Docencia Universitaria, 14(2), 1-10. https://doi.org/10.19083/ridu.2020.1315

Canaza-Choque, F. A. (2020b). La gran estampida. Humanos caminando en la modernidad líquida. Encuentros. Revista de Ciencias Humanas, Teoría Social y Pensamiento Crítico, (12), 127-145. https://doi.org/10.5281/zenodo.3951233

Canaza-Choque, F. A. (2020c). Mariátegui y la agonía de los dioses. Entre encuentros, presagios y desgracias futuras en el panteón andino. Acta Herediana, 63(2), 135-151. https://doi.org/10.20453/ah.v63i2.3834

Canaza-Choque, F. A. (2021a). «Por nuestras muertas». El despertar de un poder y la movilización de Ni Una Menos [NUM] en el Perú de 2016. PURIQ, 3(1), 22-47. https://doi.org/10.37073/puriq.3.1.107

Canaza-Choque, F. A. (2021b). El Perú de Mariátegui: detenidos en el tiempo y el despertar de una era. $\begin{array}{lllll}\text { Horizonte de la } & \text { Ciencia, } & \text { 11(20), } & \text { 45-57. }\end{array}$ https://doi.org/10.26490/uncp.horizonteciencia.2021.20.766

Canaza-Choque, F. A. (2021c). Pandemia 2020 y el poder del Estado. Daños, impactos y respuestas a zonas desprotegidas en escenarios devastadores. Socialium, 5(1), 56-74. https://doi.org/10.26490/uncp.sl.2021.5.1.752

Canaza-Choque, F. A., Supo, F., Ruelas, D., \& Yabar, P. S. (2020). El regreso del Puma Indomable. Neoliberalismo y las luchas sociales desde la escuela pública en el sur del Perú. Revista Conrado, 16(74), 154-161. Recuperado de https://conrado.ucf.edu.cu/index.php/conrado/article/view/1345

Castillo, J. M. (2016). Los negocios del cambio climático. Barcelona: Virus editorial.

Cuevas, A. B., \& Preciado, N. E. (2018). Asia Pacífico frente al Acuerdo de París contra el cambio climático: geopolítica y cooperación. Estudios de Asia y África, 53(165), 151-188. https://doi.org/10.24201/eaa.v53i1.2224

Fernández, Á. (2019). ¿Cómo afecta el cambio climático a la fauna y a la pérdida de biodiversidad? Recuperado de https://hablandoenvidrio.com/como-afecta-el- cambio-climatico-a-la-fauna-y-ala-perdida-de- biodiversidad/

García, J. (2017). Publicidad, necesidades y decrecimiento. Hacia una comunicación publicitaria menos comercial. Chasqui. Revista Latinoamericana de Comunicación, (134), 127-142. https://doi.org/10.16921/chasqui.voi134.3061

Guasch, A. (2019, 16 de septiembre). El cambio climático empuja a los animales al límite. La Vanguardia. Recuperado de https://www.lavanguardia.com/vida/juniorreport/20190916/47316764302/extincion-animales- cambio-climatico-actividad-humana.html

Hasbún-Mancilla, J. O., Aldunce-Ide, P. P., Blanco-Wells, G., \& Browne-Sartori, R. (2017). Encuadres del cambio climático en Chile: Análisis de discurso en prensa digital. Convergencia. Revista de Ciencias Sociales, 24(74), 161-186. https://doi.org/10.29101/crcs.voi74.4387

Health Effects Institute. (2020). State of Global Air 2020. Special Report. Boston: HEI.

Huanca Arohuanca, J. W. (2020a). Caleidoscopio social al Covid-19: pánico y desesperación en tiempos de aislamiento. Universidad y Sociedad, 12(6), 226-231. Recuperado de https://rus.ucf.edu.cu/index.php/rus/article/view/1836

Huanca-Arohuanca, J. W. (2020b). Cuando la muerte nos mira con deseo de llevarnos al tártaro. Revista Revoluciones, 2(2), 1-5. https://doi.org/10.35622/j.rr.2020.02.001

Huanca-Arohuanca, J. W. (2021). Narrativas de guerra y resistencia: participación de la mujer austral del Perú en la Guerra del Pacífico. Encuentros. Revista de Ciencias Humanas, Teoría Social y Pensamiento Crítico, (13), 50-59. https://doi.org/10.5281/zenodo.4395218 
Mora, C., Spirandelli, D., Franklin, E. C., Lynham, J., Kantar, M. B., Miles, W., ... Hunter, C. L. (2018). Broad threat to humanity from cumulative climate hazards intensified by greenhouse gas emissions. Nature Climate Change, (8), 1062-1071. https://doi.org/10.1038/s41558-018-0315-6

Naciones Unidas [UN]. (2019). Un millón de especies están en peligro de extinción, y la culpa es de todos. Recuperado de https://news.un.org/es/audio/2019/05/1455501

National Geographic. (2018). El cambio climático podría causar un efecto dominó en la extinción global. Recuperado de https://www.nationalgeographic.com.es/ciencia/actual idad/cambio-climaticopodria-causar-efecto-domino-extincion-global_13612

Organización de las Naciones Unidas para la Alimentación y la Agricultura [FAO]. (2020). Versión resumida de El estado de la seguridad alimentaria y la nutrición en el mundo 2020. Transformación de los sistemas alimentarios para que promuevan dietas asequibles y saludables. Roma: FAO.

Organización Mundial de la Salud [OMS]. (2018a). Cambio climático y salud. Recuperado de https://www.who.int/es/news-room/fact- sheets/detail/cambio-climático-y-salud

Organización Mundial de la Salud [OMS]. (2018b). El aire nos amenaza a todos. Recuperado de https://news.un.org/es/story/2018/05/1432612

Organización Mundial de la Salud [OMS]. (2018c, 2 de mayo). Nueve de cada diez personas de todo el mundo respiran aire contaminado. Recuperado de https://www.who.int/es/news/item/o2-052018-9-out-of-10-people-worldwide-breathe-polluted-air-but-more-countries- are-taking-action

Panel Intergubernamental del Cambio Climático [IPCC]. (2014). Cambio climático 2014: informe de síntesis. Suiza: IPCC.

Petersson, M., Marrero, M., \& Taboada, C. (2010). Cambio climático y salud humana. Rev Méd Electrón, 32(4). Recuperado de http://www.revmatanzas.sld.cu/revista medica/ano 2010/vol4 201 o/temaog.htm.

Plumer, B. (2019, 6 de mayo). La civilización acelera la extinción de más de un millón de especies y altera el mundo a un ritmo 'sin precedentes.' The New York Time. Recuperado de https://www.nytimes.com/es/2019/05/o6/civilizacio n-extincion-reporte-onu/

Rivera, J. Á. (2019). Sistemas alimentarios sostenibles para 10 mil millones de humanos en 2050. Salud Pública de México, 61(2), 104-105. https://doi.org/10.21149/10420

Sanabria, Á. (2018). La economía del carbono: una adicción de difícil tratamiento. Ciencia Política, 13(25), 51-65. https://doi.org/10.15446/cp.v12n25.69341

Sarmiento, J. P. (2018). Cuando el comercio internacional se encontró con el medio ambiente, entre el soft law y la prohibición a las barreras al comercio. ACDI-Anuario Colombiano de Derecho Internacional, 11, 187-220. https://doi.org/10.12804/revistas.urosario.edu.co/acdi/a.6542

United Nations Department of Economic and Social Affairs [UNDESA]. (2020). World Social Report 2020: Inequality in a rapidly changing world. New York: UNDESA.

Zimmer, C. (2018, 12 de diciembre). El calentamiento global ya existió... y acabó con casi toda la vida. The New York Time. Recuperado de https://www.nytimes.com/es/2018/12/12/cambio-climaticoextincion/ 